\title{
Cancer in Women
}

\author{
Aleyamma Mathew \\ Professor \& Head, Division of Cancer Epidemiology \& Bio-statistics \\ Regional Cancer Centre, Trivandrum.
}

\begin{abstract}
Cancer is emerging as a public health problem among an array of non-communicable diseases. The common cancers in women are breast, cervix uteri, colo-rectum, ovary, corpus uteri, lung and oral cavity. Breast cancer (BC) is the common cancer $(20-30 \%$ of all cancers in women) and the leading cause of cancer death in women worldwide. About half of the $\mathrm{BCs}$ and $60 \%$ of the deaths are estimated to occur in economically developing countries. In most of the registries in India, BC is the commonest cancer with the highest incidence of nearly 50 per 100,000 women in Trivandrum. Half of this cancer is reported in $<50$ years of age and it exercises adverse influence on the productive role of women in the society. The factors that contribute to the international variation in $\mathrm{BC}$ incidence rates are largely due to the differences in reproductive and hormonal factors and the availability of early detection services.
\end{abstract}

Gynecological cancers account $15-30 \%$ of all cancers in women. Cervix uteri cancer (CC) is the 3rd most common cancer affecting women worldwide, the most common cancer among women in several less developed countries and 2nd common cancer in India. During last few decades, this cancer incidence has been decreased in India. Significant declines in CC are likely due to changes in marriage and family planning, supported by underlying improvements in education and socioeconomic status. In spite of decreasing incidence of this cancer, gynecologic cancers have increased in India. Among these, ovary and corpus uteri cancers are the major contributors. Ovarian cancer (OC) has emerged as one of the common malignancies affecting women in India and is the 5th common cancer in India (4th common in Trivandrum). A steady increase has been observed in $\mathrm{OC}$ incidence in several registries including Trivandrum. More than $50 \%$ of women with OC are under the age of 50 years. The risk of it increases in

Correspondence : Dr. Aleyamma Mathew, Professor \& Head, Division of Cancer Epidemiology \& Bio-Statistics, Regional Cancer Centre, Trivandrum- 695 011. Fax: +91-471-2550782, E-mail: aleyammamathewrcc@gmail.com.

ACHANTA LAKSHMIPATHI ORATION delivered during NAMSCON 2016 at the All India Institute of Medical Sciences, Raipur. 
women who have ovulated more over their lifetime. This includes those who begin ovulation at a younger age or reach menopause at an older age. Other risk factors include hormone therapy after menopause, fertility medication and obesity. Factors that decrease risk include hormonal birth control, tubal ligation, and breast feeding. Efforts are to be made to detect ovarian cancer at an early stage by educating population about the risk factors. Corpus uteri cancers (CUC) are most common in western countries but are becoming more common in Asia. In India, the highest CUC incidence rates are observed in Trivandrum and its incidence has been increasing. Presently, it is the 5 th common cancer among women in Trivandrum, $75 \%$ of women are over the age of 50 years. The risk factors of CUC include obesity, diabetes mellitus, BC, use of tamoxifen, never having had a child, late menopause and high levels of estrogen.

Colo-rectal cancer (CRC) is the 2nd most common cancer in women world-wide. The burden of CRC has risen rapidly in some economically developed Asian countries like Japan, South Korea and Singapore. In India, the highest CRC incidence rates are observed in Trivandrum and its incidence has been increasing. Presently, it is the 5th common cancer among women in Trivandrum. The major factors include certain dietary practices and family history of cancer. Individuals with a family history of colon cancer, especially if more than one relative has had the disease, are at increased risk of CRC. Other common cancers in women are tobacco-related cancers such as oral cavity (lip, tongue and mouth) and lung. Declining trends in mouth cancer has been reported in India.

Results on the burden, trends in incidence \& mortality, risk factors of breast, cervix uteri, ovary and corpus uteri colo-rectal, lung and oral cavity cancers will be presented.

Keywords : Cancer in women, breast cancer, cervix uteri cancer, corpus uteri cancer, ovarian cancer, colo-rectal cancer, gynecological cancer, hormone therapy, menopause, oral cavity cancer.

\section{INTRODUCTION}

Cancer is emerging as a public health problem among women in an array of non-communicable diseases. Among women, it was estimated that 6.7 million new cancer cases and 3.6 million cancer deaths occurred worldwide in 2012 and common cancer sites were breast $(26 \%)$, colo-rectum (9\%), lung (9\%), cervix uteri
$(8 \%)$ and corpus uteri (5\%) (1). In India, based on the 27 cancer registries functioning under the national cancer registry programme (NCRP) of Government of India, a total of 6,95,693 women cancer cases were estimated for the year 2015 and common cancer sites were breast $(27 \%)$, cervix uteri $(23 \%)$, colo-rectum $(5 \%)$, ovary $(5 \%)$ and oral cavity (4\%). Estimated burden of these 
cancers in India were 134,214 female breast; 97,909 cervix uteri; 45,231 ovary; 25, 395 corpus uteri, 30, 309 colo-rectum; 28,542 lung and 39,090 oral cavity for the year 2015 (2). Incidence, mortality, trends and etiology of cancers such as breast, cervix uteri, ovary, corpus uteri, colorectum, lung and oral cavity are described briefly.

Breast cancer $(\mathrm{BC})$ is the most common cancer among women in both more developed (MDC) (794,000 cases) and less developed countries (LDC) (883,000 cases, among these $16.4 \%$ are from India). Age-standardized incidence rates (ASR) (per 100,000) varied nearly four-fold across the world regions, with rates ranging from 27 in Middle Africa and Eastern Asia to 96 in Western Europe (1). The rate has decreased in the US and many other developed countries since early 2000s (3). However, both incidence and mortality rates have increased in LDCs during the last two decades (4-8). More than $60 \%$ of BCs are diagnosed at the local stage in the US (SEER). Conversely, a very high proportion of cases in the developing world are detected in late stages $(9,10)$. More than $70 \%$ of $\mathrm{BC}$ patients in high-income countries are diagnosed in stages I and II whereas only $20 \%-50 \%$ patients in low- and middleincome countries are diagnosed in these earlier stages (11).

In India, BC is the commonest cancer in most of the registries with the highest incidence of nearly 50 per 100,000 women in Trivandrum. Time trends in incidence in some areas in India have increased steadily for decades, although the reasons are not well understood $(4,12$ 14). Pre-menopausal BC comprised substantially a higher proportion of all incident breast cancers in LDCs (average $47.3 \%$ ) compared to MDCs (average $18.5 \%$ ). Specifically, $48.4 \%$ of $\mathrm{BC}$ patients are diagnosed before age 50 years in India while corresponding proportions were $21.5 \%, 19.1 \%$ and $15.9 \%$ in Australia, UK and Denmark (15).

Some reproductive factors such as early age at menarche, late age at menopause, decreased total duration of breast feeding, obesity and decreased physical activity have stronger association with the risk of developing post-menopausal BC (16-21). Changes in reproductive and life-style factors mainly affect post-menopausal women whereas genetic factors probably play a more significant role in young women, though these factors alone cannot account for international variation in risk (22). Compared to post-menopausal women, $\mathrm{BC}$ in young women tend to display more aggressive features, and as a consequence a higher cause-specific mortality (23-25).

Globally, gynecological cancers account for $15-25 \%$ of all cancers in women. These cancers rank high in incidence and can affect women of all ages (26). The incidence and mortality rates of gynecologic cancers in Asian countries differ from those in Western countries (1). Cervix uteri cancer remains a major health problem in East Asia, although incidence rates have been decreasing. Behavioral factors such as 
delayed and reduced childbearing, use of hormone-replacement therapy and reduced physical activity have also become more prevalent among East Asian women (27).

Cervix uteri cancer (CC) is the 3rd most common cancer affecting women with an estimated 528,000 new cases in 2012 worldwide. A large majority (around 85\%) of the global burden occurs in the less developed regions, where it accounts for almost $12 \%$ of all female cancers. During the past few decades, incidence of this cancer has been decreased world-wide including India (28). Even though the relative importance of this disease has declined, the annual number of cases has increased. There were an estimated 266,000 deaths from CC worldwide in 2012 , accounting for $7.5 \%$ of all female cancer deaths (1). It is the 2 nd common cancer in India with highest ASR in Pazhighat $(22.5$ per 100,000$)$ followed by Mizoram $(20$ per 100,000$)$ in North east regions. However in Kerala, low incidence has been reported both in Trivandrum and Kollam (ASR: 7 per 100,000 in both areas) (2). The most important risk factor for $\mathrm{CC}$ is infection with the Human Papillomavirus (HPV). Women who smoke or whose mothers were given diethylstilboestrol during pregnancy are also at increased risk for this cancer. The decline in incidence has been attributed to a combination of factors, including improved genital hygiene, changes in marriage and family planning methods, improved treatment modalities and the beneficial effects of population-based cytological screening programmes. Screening via regular gynaecologic examinations and Pap smear followed by treatment of precancerous abnormalities decreased the incidence and mortality of CC (29).

Over the years in spite of decreasing incidence of $\mathrm{CC}$, gynecologic cancers have increased and are contributing about $30 \%$ of total cancers among women. Among these, ovary and corpus uteri cancers are the major contributors. The American Cancer Society estimated 88,750 new cases of female genital system cancers in the US in 2012 , of which 81,580 cases $(78 \%$ ) belong to the cancers of the corpus uteri $(47,130$; $53.1 \%)$ and ovary $(22,280 ; 25.1 \%)$. Estimated deaths for these cancers were 8010 and 15,500 respectively (26).

Ovarian cancer (OC) $(239,000$ cases and 152,000 deaths) is the 7 th common cancer and the 8 th cause of death from cancer in women $(3.6 \%$ of cases and $4.3 \%$ deaths). Incidence rates are high in more developed regions, with rates in these areas exceeding 15 and lowest in Sub-Saharan Africa with rates below 5 per 100,000 (1). It has emerged as one of the common malignancies affecting women in India (2). A steady increase has been observed in incidence in several cancer registries including Trivandrum in India (30). More than $50 \%$ of women with OC are under the age of 50 years. The risk of it increases in women who have ovulated more over their lifetime. Other risk factors include hormone therapy after menopause, fertility medication and obesity. Factors that decrease risk include 
hormonal birth control, tubal ligation and breast feeding $(29,31)$.

Corpus uteri cancers (CUC) are more common in western countries but are becoming common in Asia. A total of 320,000 new cases $(4.8 \%$ of cancers in women) and 76,000 deaths $(2.1 \%$ of cancer deaths in women) were reported world-wide. The highest incidence rates (ASR) were estimated in Northern America (19.1 per 100,000) and Northern and Western Europe (12.9-15.6). Rates are low in South-Central Asia (2.7) and most of Africa $(<5$ per 100,000$)$. Mortality rates ranged between 0.9 per 100,000 in Northern Africa and 3.8 per 100,000 in Melanesia (1). Increasing incidence rates of this disease have been reported in India (32). Nationally, the highest CUC incidence rates are observed in Trivandrum $(6$ per 100,000$)$. It is the 5 th common cancer among women in Trivandrum (2). Eighty percent of women with CUC are over the age of 50 years. The risk factors for it include obesity, diabetes mellitus, breast cancer, use of tamoxifen, nulliparity, late menopause and high levels of estrogen $(29,33)$.

Colo-rectal cancer (CRC) is the 2nd most common cancer in women (614,000 cases, $9.2 \%$ of the total) worldwide. There is a ten-fold geographical variation in incidence $(55 \%$ of the cases in more developed regions) across the world, the highest estimated rates per 100,000 were in Australia/New Zealand (ASR: 32.2), and the lowest in Western Africa (ASR: 3.8) (1). The burden of CRC has risen rapidly in some economically developed Asian countries (34) closing the gap to the western, industrialized populations. Economically transitioning countries have reported rapid increases in $\mathrm{CRC}$ incidence rates, with rates in the Czech Republic, Slovakia and Japan exceeded those in longstanding economically developed countries such as the United States, Canada, and Australia, these increases are thought to reflect changing dietary and physical activity patterns. In contrast, CRC rates remained stable or slightly increased from registries in majority of developed countries such as those in France, Italy, England, Germany, and Switzerland and decreased in the United States $(35,36)$.

In India, the highest CRC incidence rates (9 per 100,000) are observed in Trivandrum and its incidence has been increasing. Presently, it is the 5 th common cancer among women in Trivandrum (2). The major risk factors include certain dietary practices, reduced physical activity and family history of cancer. Individuals with a family history of colon cancer, especially if more than one relative has had the disease, are at increased risk of CRC. Time trends in incidence in some areas in India have increased steadily for decades, although the reasons are not well understood (37, 38 ). Given the large population of India, even subtle change in incidence can lead to a substantial burden of disease. CRC can often be prevented through regular screening, which can identify and remove precancerous polyps. The cause of CRC is not known, but age, presence of adenomas in the colon and rectum, family history of 
CRC in a first-degree relative, smoking and a diet high in animal fat increase the risk of developing the disease (29).

Lung cancer (LC) is the $3 \mathrm{rd}$ common cancer among women worldwide. In women, the incidence rates are generally lower and the geographical pattern is a little different, mainly reflecting different historical exposure to tobacco smoking. Thus the highest estimated age-standardized rates (per 100,000) are in Northern America (33.8) and Northern Europe (23.7) with a relatively high rate in Eastern Asia (19.2) and the lowest rates again in Western and Middle Africa (1.1 and 0.8 respectively) (1).

In India, LC was the leading site among tobacco related cancers in 11 registries among women, all the three areas of Mizoram namely, Aizawl District (40.8), Mizoram State (29.3) and Mizoram excluding Aizawl District (21.1) were at the top followed by Papumpare District (16.3) has the highest incidence rate among women (2). Rates of LC in women have been increasing since 1965, with a very modest reduction beginning only in the year 2000 (39). Smoking remains prevalent, especially in younger women (40). Smoking is the predominant cause of LC, and most cancers occur in people who smoke. In addition, nonsmoking women are more likely than men to be subject to second-hand smoke from a smoking spouse. Tobacco smoke further increases the chance of developing the disease when other environmental risk factors are present. Other substances that can cause LC, are asbestos, radon, arsenic, chromium, nickel, and tar. However, the impact of these chemicals on the incidence of LC is small compared to smoking (29).

Oral cavity cancer (OCC) accounted for 101,398 new cases in 2012 among women world-wide. The region with the highest incidence was South Central Asia (6 per 100,000) and the lowest rates in Western Asia(1.0 per $100,000)(1)$. OCC is common in India and is the 5th leading cancer among women. OCC can develop in any part of the oral cavity, the most common sites being the tongue, lip, and floor of the mouth. Rates of OCC are more than two times higher in men than in women. Declining trends in mouth cancer incidence have been in India (2) (NCRP 2016). Studies showed that tobacco use, alcohol consumption, prolonged sun exposure and infection with HPV are the factors increase the risk of developing oral cancer (29).

In conclusion, cancer is emerging as a public health problem among women. Most of the common cancers are showing an increasing trend in incidence except cancer of the cervix uteri and mouth. More than $50 \%$ of the cancers in India are reported in $<50$ years of age and mostly in late stages. Hence it exercises adverse influence on the productive role of women in the society. Public health efforts are to 
be made to understand the reasons for the striking increase in incidence of cancers and to promote education and awareness to facilitate diagnosis at earlier stage.

\section{REFERENCES :}

1. Ferlay J, Soerjomataram I, Ervik M, et al. (2014). GLOBOCAN 2012 v1.0, Cancer Incidence and Mortality Worldwide: IARC Cancer Base No. 11. Lyon, France.

2. NCRP (National Cancer Registry Programme) (2016). Three year report of the population based cancer registries 2012-2014: Report of 27 PBCRs: Indian Council of Medical Research, Bangalore, India.

3. Jemal A, Center MM, DeSantis C, Ward EM (2010). Global patterns of cancer incidence and mortality rates and trends. Cancer Epidemiol Biomarkers Prev 19:1893-1907.

4. Dhillon PK, Yeole BB, Dikshit R, et al. (2011). Trends in breast, ovarian and cervical cancer incidence in Mumbai, India over a 30-year period, 1976-2005: an age-periodcohort analysis. $\mathrm{Br} J$ Cancer $\mathbf{1 0 5}$ :723-730.

5. Lima CA, Rangel MR, MacedoLima M, da Silva AM (2012). Time trends in breast cancer incidence and mortality in a mid-sized northeastern Brazilian city. BMC Public Health 12: 883.
6. Merlo DF, Ceppi M, Filiberti R, et al. (2012). Breast cancer incidence trends in European women aged 2039 years at diagnosis. Breast Cancer Res Treat 134 :363-370.

7. Jia $\mathrm{M}$, Zheng $\mathrm{R}$, Zhang $\mathrm{S}$, et al. (2015). Female breast cancer incidence and mortality in 2011, China.J Thorac Dis 7: 1221-1226.

8. Li C, Yu C, Wang P (2015). An ageperiod-cohort analysis of female breast cancer mortality from 19902009 in China. Int $J$ Equity Health 14:76.

9. Sloan FA, Gelband H, editors ( 20007 ). Cancer Control Opportunities in Low- and MiddleIncome Countries. Washington, DC, USA: Institute of Medicine of the National Academies.

10. Porter P (2008). "Westernizing" women's risks? Breast cancer in lower-income countries. NEJM 358 :213-216.

11. Unger-Saldaña K ( 201014$)$. Challenges to the early diagnosis and treatment of breast cancer in developing countries. World J Clin Onco 110: 465-477.

12. Murthy NS, Shalini S, Suman G, Pruthvish S, Mathew A (2009). Changing trends in incidence of ovarian cancer - the Indian scenario. Asian Pacific $J$ Cancer Prev 10: 1025-1030. 
13. Dikshit RP, Yeole BB, Nagrani R, et al. (2012). Increase in breast cancer incidence among older women in Mumbai: 30-year trends and predictions to 2025. Cancer Epidemiol 36: e215-e220.

14. Mathew A, George PS, Arjunan A, et al. (2016). Temporal Trends and Future Prediction of Breast Cancer Incidence across Age Groups in Trivandrum, South India. APJCP 17: 2895-2899.

15. Ghiasvand R, Adami H-O, Harirchi I, et al. (2014). Higher incidence of premenopausal breast cancer in less developed countries; myth or truth? BMC Cancer 14: 343.

16. Mathew A, Gajalakshmi V, Rajan B, et al. (2008). Pattern of anthropometric factors among urban and rural women in south India and the risk of breast cancer: a multicentric case-control study. $\mathrm{Br} J$ Cancer 99: 207-213.

17. Mathew A, Gajalakshmi V, Rajan B, et al. (2009). Physical activity level among urban and rural women in south India and the risk of breast cancer: a case-control study. Euro $J$ Cancer Prev 18: 368-376.

18. Gajalakshmi V, Mathew A, Brennan $\mathrm{P}$, et al. (2009). Breastfeeding and breast cancer risk in India: a multicenter case-control study. Int $J$ Cancer 125: 662-665.
19. Rose DP, Vona-Davis L (2010). Interaction between menopausal status and obesity in affecting breast cancer risk. Maturitas 66: 33-38.

20. Hemminki K, Försti A, Sundquist J, Mousavi S (2011). Preventable breast cancer is postmenopausal. Breast Cancer Res Treat 125: 163-167.

21. Amadou A, Ferrari P, Muwonge R, et al. (2013). Overweight, obesity and risk of premenopausal breast cancer according to ethnicity: a systematic review and doseresponse meta-analysis. Obes Rev 14: 665-678.

22. Assi HA, Khoury KE, Dbouk H, et al. (2013). Epidemiology and prognosis of breast cancer in young women. J Thorac Dis 5: S2-S8.

23. Mathew A, Rajan B, Pandey M (2004). Do younger women with non-metastatic and noninflammatory breast carcinoma have poor prognosis? World J Surg Oncol 2: 2 .

24. Anders CK, Johnson R, Litton J, et al. (2009). Breast Cancer before Age 40 Years. Semin Oncol 36: 237-249.

25. Keramatinia A, Mousavi-Jarrahi S, Hiteh M, Mosavi-Jarrahi A (2014). 
Trends in Incidence of Breast Cancer among women under 40 in Asia. Asian Pac J Cancer Prev 15: 1387-1390.

26. Forouzanfar $\mathrm{MH}$, Foreman $\mathrm{KJ}$, Delossantos AM, et al. (2011). The accuracy of cancer mortality statistics based on death certificates in the United States. Cancer Epidemiol 35(2):126-131.

27. Katanoda K, Matsuda T, Matsuda A, et al. (2013). An updated report of the trends in cancer incidence and mortality in Japan. Jpn J Clin Oncol 43: 492-507.

28. Mathew A, George PS (2009). Trends in incidence and mortality rates of squamous cell carcinoma and adenocarcinoma of cervix -worldwide. Asian Pacific J Cancer Prev 10 (4): 645-650.

29. Morris CR, Epstein J, Nassere K, et al. (2010). Trends in Cancer Incidence, Mortality, Risk Factors, and Health Behaviors in California. Sacramento, CA: California Department of Public Health, Cancer Surveillance Section.

30. Murthy NS, Chaudhry K, Nadayil $\mathrm{D}$, et al. (2009). Changing trends in incidence of breast cancer: Indian scenario. Ind J Cancer 46: 73-74.
31. Pasalich M, Su D, Binns CW, Lee AH (2013). Reproductive factors for ovarian cancer in southern Chinese women. J Gynecol Oncol 24:135140.

32. Murthy NS, Shalini S, Sastry NB, Suman G, Sreekantaiah P, Mathew A (2011). Increase in incidence of cancer of corpus uteri: estimation of time trends - an Indian scenario. Eur JCancer Prev 20:25-32.

33. Cramer DW ( $\left.\begin{array}{llll}2 & 0 & 1 & 2\end{array}\right)$. The epidemiology of endometrial and ovarian cancer. Hematol Oncol Clin North Am 26:1-12.

34. Kuriki K, Tajima K (2006). The increasing incidence of colorectal cancer and the preventive strategy in Japan. Asian Pac J Cancer Prev 7: 495-501.

35. Plesko I, Boyle GS, Ondrusova M, Tomasek L, Kubik A (2008). Dominant position of colorectal cancer in Slovakia: the old-new problem for cancer control. Neoplasma 55:10-15.

36. Center MM, Jemal A, Ward E (2009). International trends in colorectal cancer incidence rates. Epidemiol Biomarkers Prev 18(6):1688-1694. 
37. Yeole BB (2008). Trends in Cancer Incidence in Esophagus, Stomach, Colon, Rectum and Liver in Males in India. Asian Pacific J Cancer Prev 9: 97-100.

38. Nooyi SC, Murthy N S, Shivananjaiah S, Sreekantaiah $\mathrm{P}$, Mathew A (2011). Trends in Rectal Cancer Incidence - Indian Scenario. Asian Pacific J Cancer Prev 12:2001-2006.
39. Siegel R, Ward E, Brawley O, Jemal A (2011).The impact of eliminating socioeconomic and racial disparities on premature cancer deaths. $C A$ Cancer J Clin 61(4):212-236.

40. Patel JD (2005). Lung cancer in women. J Clin Oncol $\mathbf{2 3}$ :3212-3218. 